\title{
Attitude Estimation Based on Time-Varying Reference Vectors with Biased Gyro and Vector Measurements *
}

\author{
Håvard Fjær Grip * Thor I. Fossen ${ }^{* *}$ Tor A. Johansen ${ }^{* *}$ Ali Saberi ${ }^{*}$ \\ * School of Electrical Engineering and Computer Science, Washington State \\ University, Pullman, WA 99164-2752, USA \\ ** Department of Engineering Cybernetics, Norwegian University of Science \\ and Technology, NO-7491 Trondheim, Norway
}

\begin{abstract}
We present two results on attitude estimation using vector and rate gyro measurements, when both sets of measurements are biased. The first result concerns an observer for attitude and gyro bias that has previously been presented by Hamel and Mahony, and by Mahony, Hamel, and Pflimlin, with proven almost-global stability results when either (i) the reference vectors in the inertial frame are stationary; or (ii) the reference vectors are time-varying but the gyro measurements are unbiased. We prove that the same observer with an added parameter projection is semiglobally exponentially stable when bias estimation is included and the reference vectors are time-varying. The second result concerns estimation of bias in the body-fixed vector measurements. We show how the bias can be estimated in a manner that is decoupled from the attitude and gyro bias estimation, provided the measurements are sufficiently excited relative to the level of measurement noise.
\end{abstract}

Keywords: navigation; nonlinear observers; sensor data fusion

\section{INTRODUCTION}

Determination of the attitude of a vehicle with respect to a reference frame is one of the fundamental tasks of a navigation system. Frequently, the attitude is determined by comparing vectors measured in a body-fixed coordinate frame with known reference vectors in the reference frame. The necessary vector measurements can be obtained, for example, by using on-board magnetometers, which measure the earth's magnetic field; star-trackers, which measure the position of stars relative to the vehicle; or accelerometers, which measure the earth's gravity field together with the acceleration of the vehicle. If at least two non-parallel vector measurements and their corresponding reference vectors are available, then the attitude can be determined by using algorithms like the QUEST algorithm (Shuster and Oh, 1981).

In practice, vector measurements are affected by noise, which is particularly significant at high frequencies. It is therefore common to combine vector measurements with measurements from rate gyroscopes, which complement the vector measurements by providing high-frequency information about the attitude.

Most commonly, vector and gyroscope measurements are integrated using an extended Kalman filter (EKF). An overview of early EKF implementations is given by Lefferts, Markley, and Shuster (1982). Crassidis, Markley, and Cheng (2007) survey more recent results based on EKFs, as well as other estimation techniques such as unscented filtering, particle filtering, nonlinear observers, and extensions of the QUEST

\footnotetext{
^ The work of Håvard Fjær Grip is supported by the Research Council of Norway. The work of Ali Saberi is partially supported by NAVY grants ONR KKK777SB001 and ONR KKK760SB0012.
}

algorithm. Integrated inertial (INS) and satellite (GNSS) navigation systems have also been studied for several decades and are typically based on EKFs (Maybeck, 1979; Phillips and Schmidt, 1996; Grewal, Weill, and Andrews, 2001). Such systems implicitly determine the attitude by matching the accelerometer vector with changes in the velocity vector.

One alternative to the EKF is to construct nonlinear observers with explicitly proven stability properties, by using, for example, Lyapunov-based methods. Such observers typically have a smaller computational footprint than EKF-based solutions, and they are therefore of particular interest as low-cost navigation equipment becomes available in an increasingly wide range of products, such as cell phones, cars, and small unmanned vehicles.

The first observer of this type was presented by Salcudean (1991). Salcudean's observer was later extended by Vik and Fossen (2001), by adding gyro bias estimation as well as linear velocity estimation in the context of GPS/INS integration. Thienel and Sanner (2003) improved the stability analysis of Vik and Fossen (2001) by deriving a persistency-of-excitation condition that guarantees exponentially vanishing estimation errors. The nonlinear observers based on Salcudean's work assume that the attitude is resolved in a separate subsystem and provided as a measurement to the observer. A drawback of this assumption is that the noise characteristics of the resolved attitude may differ significantly from the characteristics of the measurements used to produce it.

More recently, Hamel and Mahony (2006); Mahony, Hamel, and Pflimlin (2008) have proposed an explicit complementary filter that does not rely on a separate subsystem for resolving the attitude, but instead makes use of vector measurements directly. Their observer includes estimation of gyro bias and 
is rigorously analyzed with respect to stability. An underlying assumption, however, is that the reference vectors are stationary (constant). Stability in the case of time-varying reference vectors is studied in a later paper by Mahony, Hamel, Trumpf, and Lageman (2009) and in a recent paper by Hua (2010), albeit without considering gyro bias estimation. It is proven that the observer without bias estimation can in fact handle time-varying reference vectors as well. Moreover, Hua presents algorithms for using the derivative of GNSS velocity as a reference vector without explicit differentiation.

\subsection{Contributions}

This paper is concerned with two problems on the topic of attitude and bias estimation using nonlinear observers. First, we consider the observer by Hamel and Mahony (2006); Mahony et al. (2008). As discussed above, this observer has proven stability properties when either (i) the reference vectors are stationary; or (ii) the reference vectors are timevarying but the gyro measurements are bias-free. What is not considered by Hamel and Mahony (2006), Mahony et al. (2008, 2009) or Hua (2010) is the case when the reference vectors are time-varying and the gyro measurements are biased. By adding a parameter projection to the bias estimation algorithm, we show in Section 5 that the observer is applicable in this case as well; in particular, we prove semiglobal exponential stability for this case.

In addition to gyro bias, bias in the vector measurements can constitute a significant problem. A particular example is when an accelerometer measurement is used as one of the body-fixed vector measurements. Low-cost accelerometers typically have a large bias; on the other hand, the corresponding reference vector, derived using the gravity vector and possibly the derivative of GNSS velocity, is typically subject to a very small bias. Motivated by this situation, we present in Section 6 an algorithm for estimating bias in the body-fixed vector measurement by using a bias-free reference vector. The algorithm is decoupled from the attitude and gyro bias estimation, and it is therefore analyzed on its own.

\section{PRELIMINARIES}

Any rotation can be described by a unit vector $k \in \mathbb{R}^{3}$ about which the rotation takes place, and an angle of rotation $\beta$. The corresponding unit quaternion $q$ is defined as $q=\left[s, r^{\top}\right]^{\top}=$ $\left[\cos (\beta / 2), k^{\top} \sin (\beta / 2)\right]^{\top}$, which implies $\|q\|^{2}=s^{2}+\|r\|^{2}=1$. We shall alternately describe rotations by unit quaternions and rotation matrices $R \in \mathrm{SO}(3)$. The two representations are related by $R=R_{q}(s, r):=I+2 s S(r)+2 S(r)^{2}$, where $S(x)$ denotes the skew-symmetric matrix such that for all $x, y \in \mathbb{R}^{3}, S(x) y=x \times y$. For all $x, y \in \mathbb{R}^{3}$ and all $R \in \mathrm{SO}(3)$, the following holds for $S(\cdot): S(x)=-S(x)^{\top} ; y^{\top} S(x) y=0$; and $R S(x) R^{\top}=S(R x)$. We refer to Fossen (2011) for more detailed background information.

We denote by $\|\cdot\|$ the Euclidean norm for vectors in $\mathbb{R}^{n}$ and the Frobenius norm for matrices in $\mathbb{R}^{m \times m}$. For $(X, x) \in$ $\mathbb{R}^{m \times m} \times \mathbb{R}^{n}$, we define $\|(X, x)\|=\sqrt{\|X\|^{2}+\|x\|^{2}}$. For a symmetric matrix $X$ we denote by $\lambda_{\min }(X)$ and $\lambda_{\max }(X)$ the minimum and maximum eigenvalues of $X$. Throughout the paper, we assume that the various dynamical systems are initialized at time $t=0$. For time-varying variables, we shall omit the time argument unless it is explicitly needed.

\section{PROBLEM FORMULATION}

Let $R \in \mathrm{SO}$ (3) denote the rotation matrix from the body-fixed frame to an inertial reference frame. The dynamics of $R$ is described by

$$
\dot{R}=R S(\omega),
$$

where $\omega$ represents the angular velocity of the vehicle body in body-fixed coordinates. The angular velocity is presumed bounded by $\|\omega\| \leq \bar{\omega}$. A measurement $\omega_{m}=\omega+b_{g}$ is available, where $b_{g}$ represents gyro bias. We assume that $b_{g}$ is constant and belongs to an a priori known, compact, convex set $\mathcal{B}_{g} \subset \mathbb{R}^{3}$. This assumption is reasonable, as it only requires knowledge of some bound on the magnitude $\left\|b_{g}\right\|$.

We furthermore assume availability of $n$ vectors $v_{j}^{b}, j \in$ $1, \ldots, n$, in the body-fixed frame, as well as corresponding reference vectors $v_{j}^{i}=R v_{j}^{b}, j \in 1, \ldots, n$, in the inertial frame. The reference vectors are presumed to be time-varying but always nonzero. Unless otherwise stated, we also assume that the vector measurements have been normalized so that $\left\|v_{j}^{b}\right\|=\left\|v_{j}^{i}\right\|=1$. As in other places in the literature (Mahony et al., 2009; Hua, 2010), we ensure that the attitude is observable through an additional assumption regarding the reference vectors.

Assumption 1. There exists a constant $c_{\mathrm{obs}}>0$ such that, for each $t \geq 0$, the inequality $\left\|v_{j}^{i} \times v_{k}^{i}\right\| \geq c_{\text {obs }}$ holds for some pair of indices $j, k \in 1, \ldots, n$.

Remark 1. Practically speaking, Assumption 1 requires there to always be two normalized reference vectors with the angle between them bounded away from $0^{\circ}$ and $180^{\circ}$.

\subsection{Attitude Error Representation}

The estimate of the rotation matrix will be denoted by $\hat{R}$, and the attitude estimation error will be represented by a rotation matrix $\tilde{R}:=R \hat{R}^{\top}$. The goal is to ensure that $\tilde{R} \rightarrow I$ as $t \rightarrow \infty$, which implies that $\hat{R}$ asymptotically represents the same rotation as $R$. The attitude error will also be represented by a unit quaternion $\tilde{q}=\left[\tilde{s}, \tilde{r}^{\top}\right]^{\top}$ corresponding to $\tilde{R}$. Note that $\tilde{R}=I$ is equivalent to $|\tilde{s}|=1$ and $\tilde{r}=0$. It is also useful to note that $\tilde{s}=0$ corresponds to a rotation error of $180^{\circ}$ about some axis (i.e., the maximal error rotation angle).

\section{PREVIOUS RESULTS}

Hamel and Mahony (2006); Mahony et al. (2008) present the following observer for estimating $R$ and $b_{g}$ :

$$
\begin{aligned}
\dot{\hat{R}} & =\hat{R} S\left(\omega_{m}-\hat{b}_{g}+\sigma\right), \\
\dot{\hat{b}}_{g} & =-k_{I} \sigma,
\end{aligned}
$$

where $\sigma$ represents an injection term given by

$$
\sigma=\sum_{j=1}^{n} k_{j} v_{j}^{b} \times \hat{R}^{\top} v_{j}^{i},
$$

with $k_{j} \geq k_{P}>0, i=1, \ldots, n$, as well as $k_{I}$, representing scalar observer gains. Under the assumption that the reference vectors $v_{j}^{i}, j=1, \ldots, n$, are stationary, it is proven that the observer states converge to the true states for almost all initial conditions.

\subsection{Time-Varying Reference Vectors}

If the reference vectors $v_{j}^{i}, j=1, \ldots, n$, are time-varying, then convergence of $\hat{R}$ to $R$ can still be proven if the presence of 
gyro bias is disregarded (i.e., $\omega_{m}=\omega$ and $\hat{b}_{g}=0$ ). This can be done, for example, based on the analysis of Hua (2010), as outlined in the remainder of this section.

The dynamics of the error variable $\tilde{R}$ is described by $\dot{\tilde{R}}=$ $\dot{R} \hat{R}^{\top}+R \dot{\hat{R}}^{\top}=R S(\omega) \hat{R}^{\top}-R S(\omega+\sigma) \hat{R}^{\top}=-R S(\sigma) \hat{R}^{\top}=$ $-S(R \sigma) \tilde{R}$, where $S(x)^{\top}=-S(x)$ and $R S(x) R^{\top}=S(R x)$ have been used. The dynamics described by a corresponding unit quaternion is therefore (Fossen, 2011)

$$
\dot{\tilde{s}}=\frac{1}{2} \tilde{r}^{\top} R \sigma, \quad \dot{\tilde{r}}=-\frac{1}{2}(\tilde{s} I-S(\tilde{r})) R \sigma .
$$

The unit quaternion dynamics is confined to the unit sphere; that is $\tilde{s}+\|\tilde{r}\|^{2}=1$. Note that

$$
\begin{aligned}
R \sigma & =\sum_{j=1}^{n} k_{j} R S\left(v_{j}^{b}\right) \hat{R}^{\top} v_{j}^{i}=\sum_{j=1}^{n} k_{j} S\left(R v_{j}^{b}\right) \tilde{R} v_{j}^{i} \\
& =\sum_{j=1}^{n} k_{j} S\left(v_{j}^{i}\right)\left(I+2 \tilde{s} S(\tilde{r})+2 S(\tilde{r})^{2}\right) v_{j}^{i} \\
& =2 \sum_{j=1}^{n} k_{j} S\left(v_{j}^{i}\right)\left(\tilde{s} S(\tilde{r})+S(\tilde{r})^{2}\right) v_{j}^{i} .
\end{aligned}
$$

By defining the Lyapunov-like function

$$
V(\tilde{s})=1-\tilde{s}^{2}=\|\tilde{r}\|^{2},
$$

one obtains the time derivative

$$
\begin{aligned}
\dot{V} & =-2 \tilde{s} \tilde{r}^{\top} \sum_{j=1}^{n} k_{j} S\left(v_{j}^{i}\right)\left(\tilde{s} S(\tilde{r})+S(\tilde{r})^{2}\right) v_{j}^{i} \\
& =-2 \tilde{s} \sum_{j=1}^{n} k_{j}\left(\tilde{r} \times v_{j}^{i}\right)^{\top}(\tilde{s} I+S(\tilde{r}))\left(\tilde{r} \times v_{j}^{i}\right) \\
& \leq-2 k_{P} \sum_{j=1}^{n} \tilde{s}^{2}\left\|\tilde{r} \times v_{j}^{i}\right\|^{2},
\end{aligned}
$$

where we have used the identity $y^{\top} S(x) y=0$. Finally, using the inequality $\left\|\tilde{r} \times v_{j}^{i}\right\|^{2}+\left\|\tilde{r} \times v_{k}^{i}\right\|^{2} \geq \frac{1}{2}\|\tilde{r}\|^{2}\left\|v_{j}^{i} \times v_{k}^{i}\right\|^{2}$ (Hua, 2010) together with Assumption 1 yields

$$
\dot{V} \leq-k_{P} c_{\mathrm{obs}}^{2} \tilde{s}^{2}\|\tilde{r}\|^{2}=-k_{P} c_{\mathrm{obs}}^{2} \tilde{s}^{2}\left(1-\tilde{s}^{2}\right) .
$$

Since $\dot{V}$ is negative whenever $\tilde{s} \neq 0$ and $|\tilde{s}| \neq 1$, it is straightforward to show that for any initial condition satisfying

$$
\tilde{R}(0) \in\left\{R_{q}(\tilde{s}, \tilde{r})|| \tilde{s} \mid>0\right\} .
$$

$|\tilde{s}|$ will increase monotonically such that $|\tilde{s}| \rightarrow 1$ (implying $\|\tilde{r}\| \rightarrow 0$ and $\tilde{R} \rightarrow I$ ). That is, the initial rotation error must be strictly less than $180^{\circ}$.

\section{ATTITUDE AND GYRO BIAS ESTIMATION WITH TIME-VARYING REFERENCE VECTORS}

In this section we build on the analysis outlined in the previous section to show that the observer, including bias estimation, is applicable even if the reference vectors are time-varying. The only modification that we make to (2) is to add a parameter projection of the type shown in Appendix A (Krstić, Kanellakopoulos, and Kokotović, 1995, App. E), which ensures that $\hat{b}_{g}$ remains within a compact, convex set $\hat{\mathcal{B}}_{g} \supset \mathcal{B}_{g}$, defined slightly larger than $\mathcal{B}_{g}$. Accordingly, we rewrite $(2 \mathrm{~b})$ as

$$
\dot{\hat{b}}_{g}=\operatorname{Proj}\left(\hat{b}_{g},-k_{I} \sigma\right) \text {. }
$$

Defining the bias estimation error $\tilde{b}_{g}=b_{g}-\hat{b}_{g}$, we obtain the complete error dynamics

$$
\dot{\tilde{R}}=-S\left(R \tilde{b}_{g}+R \sigma\right) \tilde{R}, \quad \dot{\tilde{b}}_{g}=-\operatorname{Proj}\left(\hat{b}_{g},-k_{I} \sigma\right) .
$$

To state the stability result for (8), we introduce the parameterized set $\tilde{\mathcal{R}}(\varepsilon)=\left\{R_{q}(\tilde{s}, \tilde{r})|| \tilde{s} \mid \geq \varepsilon\right\}$, where $0<\varepsilon<1$.

Theorem 1. Consider the dynamics of $\left(\tilde{R}, \tilde{b}_{g}\right) \in \mathrm{SO}(3) \times \mathbb{R}^{3}$. For each $0<\varepsilon<1$, there exists a $\bar{k}_{P}>0$ such that, for all $k_{P}>\bar{k}_{P}$, the equilibrium point $(I, 0)$ is exponentially stable with all initial conditions such that $\tilde{R}(0) \in \tilde{\mathcal{R}}(\varepsilon)$ and $\hat{b}_{g}(0) \in \hat{\mathcal{B}}_{g}$ contained in the region of attraction.

The proof of Theorem 1 is given in Appendix B. Compared to (6), we see that the requirement on the initial condition $\tilde{R}(0)$ in Theorem 1 specifies that the initial error rotation angle must not only be strictly smaller than $180^{\circ}$, but smaller by a certain margin. This margin can be made arbitrarily small by increasing $k_{P}$. In addition, the initial bias estimate must be initialized from the set $\hat{\mathcal{B}}_{g}$, which has been chosen to encompass all possible values of the actual bias $b_{g}$. The stability result is therefore best described as semiglobal (in the same way as Hua, 2010), which is slightly weaker than the almost-global result for the case of stationary reference vectors (Mahony et al., 2008).

When estimating constant parameters using Lyapunov-based techniques, it is common for the derivative of the Lyapunov function to become negative semidefinite rather than negative definite, thus requiring a further invariance-based argument to prove asymptotic stability (e.g., based on Barbălat's lemma or LaSalle's invariance principle). By contrast, the proof of Theorem 1 provides a Lyapunov function with a negative definite derivative within the region of attraction. The availability of such a Lyapunov function is often an advantage, as it provides an explicit stability margin that can be useful, for example, when studying robustness or interconnections with other subsystems.

The observer can be implemented in terms of unit quaternions instead of rotation matrices, as described by Mahony et al. (2008, App. B). This is generally more desirable, as it involves a four-dimensional vector of unit length rather than a $3 \times 3$ matrix in $\mathrm{SO}(3)$.

\section{ESTIMATION OF VECTOR BIAS}

A potentially significant problem that is not accounted for in the observer (2) is the presence of bias in the vector measurements. As mentioned in Section 1.1, a particular situation where vector bias may pose a problem is when an accelerometer measurement is used as one of the body-fixed vectors. Motivated by this scenario, we consider a single, biased vector measurement $v_{m}^{b}=v^{b}+b_{v}$ in the body-fixed frame, where $v^{b}$ is the true value of the vector and $b_{v}$ is a constant bias. By using the unbiased reference vector measurement $v_{m}^{i}=v^{i}=$ $R v^{b}$, we shall estimate the bias $b_{v}$ asymptotically. Moreover, we shall do so in a way that is completely independent of the attitude or the gyro bias, meaning that the vector bias estimation can be studied separately from the attitude and gyro bias estimation. In this section we no longer assume that $v^{b}$ and $v^{i}$ are normalized to have magnitude 1 .

Define $y=\left\|v_{m}^{i}\right\|^{2}-\left\|v_{m}^{b}\right\|^{2}$ and $p=\left\|b_{v}\right\|^{2}$. Noting that $\left\|v^{i}\right\|=\left\|R v^{b}\right\|=\left\|v^{b}\right\|$, we calculate

$$
\begin{aligned}
y & =\left\|v^{i}\right\|^{2}-\left\|v^{b}+b_{v}\right\|^{2}=\left\|v^{i}\right\|^{2}-\left\|v^{b}\right\|^{2}-\left\|b_{v}\right\|^{2}-2\left(v^{b}\right)^{\top} b_{v} \\
& =-\left\|b_{v}\right\|^{2}-2\left(v^{b}+b_{v}\right)^{\top} b_{v}+2 b_{v}^{\top} b_{v} \\
& =\left\|b_{v}\right\|^{2}-2\left(v_{m}^{b}\right)^{\top} b_{v}=p-2\left(v_{m}^{b}\right)^{\top} b_{v} .
\end{aligned}
$$


Defining the constant vector $\theta=\left[p, b_{v}^{\top}\right]^{\top}$ and the timevarying vector $\phi=\left[1,-2\left(v_{m}^{b}\right)^{\top}\right]^{\top}$, we can now write

$$
y=\phi^{\top} \theta \text {. }
$$

Based on (9), we may construct an over-parameterized observer

$$
\dot{\hat{\theta}}=\Gamma \phi\left(y-\phi^{\top} \hat{\theta}\right),
$$

where $\Gamma$ is a symmetric, positive-definite gain matrix. Defining the error variable $\tilde{\theta}=\theta-\hat{\theta}$, the error dynamics becomes

$$
\dot{\tilde{\theta}}=-\Gamma \phi \phi^{\top} \tilde{\theta} \text {. }
$$

The following theorem gives a stability result for (11), subject to a persistency-of-excitation condition.

Theorem 2. Suppose that there exist $\varepsilon>0$ and $T>0$ such that, for each $t \geq 0$,

$$
\int_{t}^{t+T} \phi(\tau) \phi^{\top}(\tau) \mathrm{d} \tau \geq \varepsilon I
$$

Then the origin is a globally exponentially stable equilibrium point for (11).

Proof. See Appendix B.

The requirement (12) is a standard persistency-of-excitation condition that can be interpreted as requiring the individual components of $\phi$ (consisting of a constant 1 and the elements of the vector $-2 v_{m}^{b}$ ) to vary in a sufficiently independent manner. Whether this requirement is reasonable or not depends on the specifics of the application.

Remark 2. Equation (9) represents a linear regression model, with output $y$, regressor $\phi$, and parameter vector $\theta$. Several standard estimation methods can therefore be applied. For example, by introducing a time-varying gain $\Gamma$ satisfying $\dot{\Gamma}=$ $\alpha \Gamma-\Gamma \phi \phi^{\top} \Gamma$, with $\alpha \geq 0$, we obtain the recursive leastsquares estimate of $\theta$ with forgetting factor $\alpha$ (Åström and Wittenmark, 1995, Th. 2.5).

If the vector bias estimation is combined with the attitude and gyro bias estimation in Section 5, then the overall error dynamics becomes a cascade interconnection where the attitude and gyro bias error is affected by the vector bias error, but not the other way around. Analysis of cascade interconnections is often straightforward (see, e.g., Panteley and Loría, 2001), because the states of the second subsystem in the cascade can often be shown to converge to the origin when affected by a vanishing disturbance from the first subsystem. Due to the semiglobal nature of Theorem 1, however, the attitude estimate may be pushed out of the region of attraction if the initial vector bias is large. Therefore, only a regional stability result can currently be proven for the interconnected system. Simulation results nevertheless indicate that this is not a practical problem.

\section{SIMULATION}

In this section we consider a simulation example where two vector measurements in the body-fixed frame and their corresponding reference vectors are available. The first of these vector measurements is a biased accelerometer measurement, with a corresponding reference vector representing the acceleration of the body in the inertial frame minus the gravity vector (obtained, for example, by differentiating GNSS velocity). The second vector measurement has a stationary reference vector and may represent, for example, a magnetometer measurement. The motion in this example is not

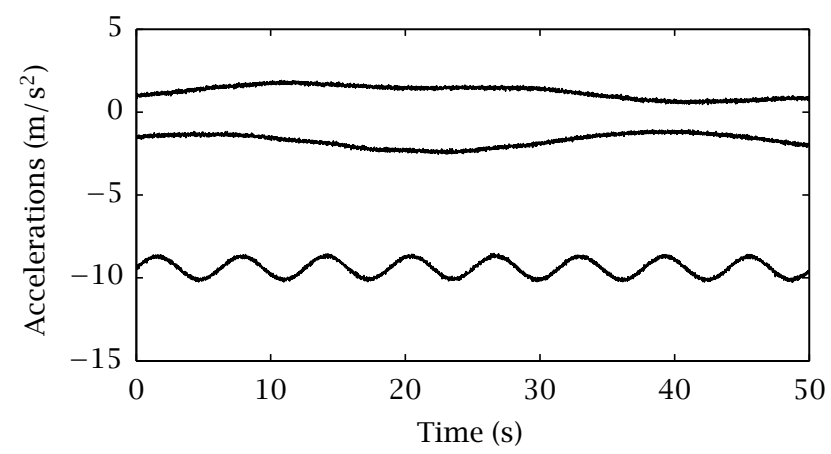

Fig. 1. Accelerometer measurement

constructed to correspond to a physically realistic situation, as the goal is merely to study the response of the estimation algorithms to a particular excitation.

The accelerometer measurements, including bias, are shown for the first $50 \mathrm{~s}$ in Fig. 1. The variation in the acceleration measurements are due to a combination of body acceleration and changes in the attitude (resulting in variation in the direction of the measured gravity vector). The same motion pattern is repeated throughout the simulation. Band-limited white noise has been added to the accelerometer measurements, as well as the other body-fixed vector measurement, the reference vectors, and the gyroscope measurement. Before being used in any of the estimation algorithms, the measurements are filtered using first-order low-pass filters with a $5-\mathrm{Hz}$ cut-off frequency.

The vector bias estimation is implemented using the timevarying gain corresponding to the recursive least-squares algorithm, as described in Remark 2, with initial gain $\Gamma(0)=$ $10 I$ and forgetting factor $\alpha=0$. The attitude and gyro bias observer with parameter projection is implemented with gains $k_{1}=k_{2}=0.5$ and $k_{I}=0.1$.

The estimates of the accelerometer bias, attitude, and gyro bias are shown, together with the actual values, in Fig. 2. With the chosen gains, it takes a considerable amount of time for the estimates to converge. The convergence time can be reduced at the expense of increased sensitivity to noise.

\subsection{Estimation Bias}

In the presence of measurement noise, the estimate of $\theta$ is not an unbiased one. The first reason for this is that, by using $\left\|v_{m}^{i}\right\|^{2}$ and $\left\|v_{m}^{b}\right\|^{2}$ to produce $y$, the squared norm of the noise components of $\left\|v_{m}^{i}\right\|$ and $\left\|v_{m}^{b}\right\|$ are included in $y$, and these have a nonzero expected value. This does not constitute a major problem, as it effectively introduces an extra constant term that is additive with the redundant parameter $p$. The second reason is that the regressor $\phi$ contains noise, and it is well-known that noisy regressors generally lead to biased estimates (Hong, Söderström, and Zheng, 2007). The lowpass filtering mentioned above is crucial to reducing this effect; if the noise level is too large relative to the level of excitation, the estimates become severely degraded, which may be detrimental in many applications. Other options for reducing the estimation bias include compensation by using a priori knowledge of the regressor noise intensity; and the introduction of additional parameters (Hong et al., 2007). 


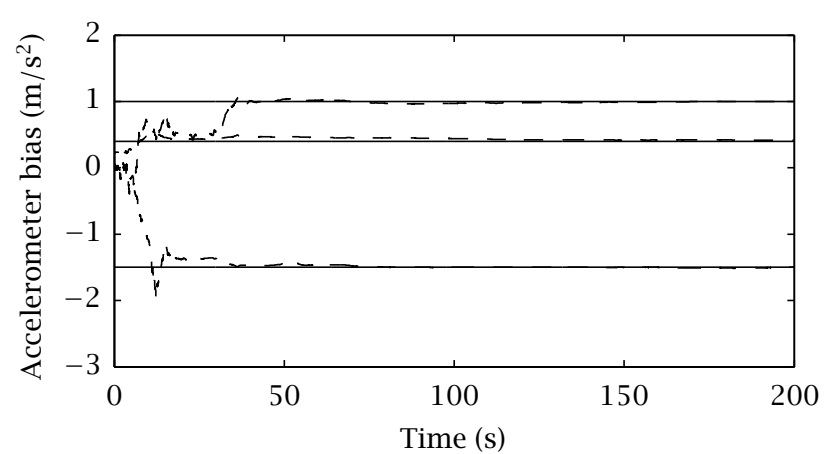

(a) Actual (solid) and estimated (dashed) accelerometer bias

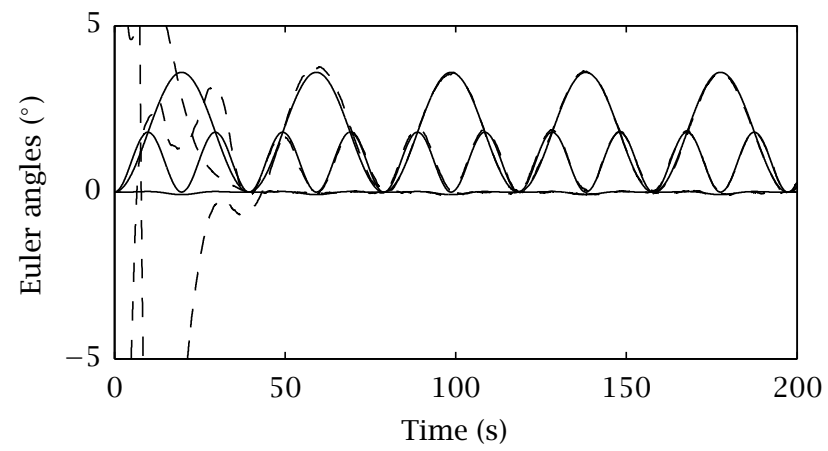

(b) Actual (solid) and estimated (dashed) Euler angles

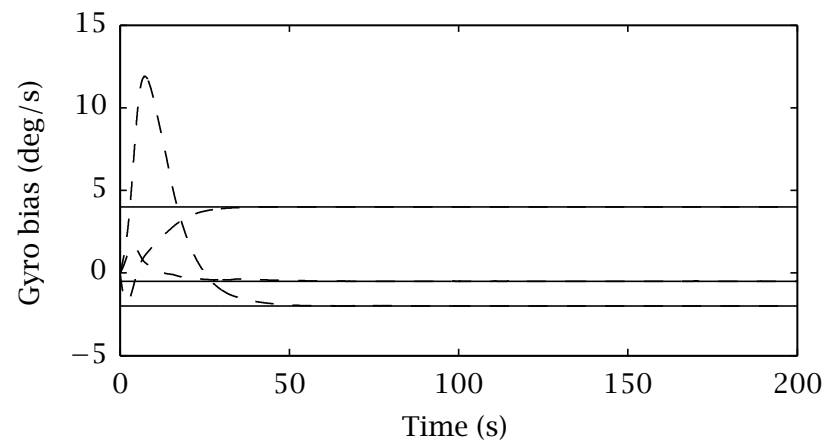

(c) Actual (solid) and estimated (dashed) gyro bias

Fig. 2. Simulation results

\section{ACKNOWLEDGMENTS}

The authors would like to thank Dr. Bjørnar Vik for many valuable discussions.

\section{REFERENCES}

Åström, K. J., Wittenmark, B., 1995. Adaptive Control, 2nd Edition. Addison-Wesley, Reading, MA.

Crassidis, J. L., Markley, F. L., Cheng, Y., 2007. Survey of nonlinear attitude estimation methods. J. Guid. Contr. Dynam. 30 (1), 12-28.

Fossen, T. I., 2011. Handbook of Marine Craft Hydrodynamics and Motion Control. Wiley.

Grewal, M., Weill, L., Andrews, A., 2001. Global Positioning Systems, Inertial Navigation, and Integration. Wiley.

Hamel, T., Mahony, R., 2006. Attitude estimation on SO(3) based on direct inertial measurements. In: Proc. IEEE Int Conf. Robotics Automation. Orlando, FL, pp. 2170-2175.

Hong, M., Söderström, T., Zheng, W. X., 2007. Accuracy analysis of bias-eliminating least squares estimates for errorsin-variables systems. Automatica 43, 1590-1596.
Hua, M.-D., 2010. Attitude estimation for accelerated vehicles using GPS/INS measurements. Contr. Eng. Pract. 18 (7), 723-732.

Khalil, H. K., 2002. Nonlinear Systems, 3rd Edition. PrenticeHall, Upper Saddle River, NJ.

Krstić, M., Kanellakopoulos, I., Kokotović, P. V., 1995. Nonlinear and Adaptive Control Design. Wiley, New York.

Lefferts, E. J., Markley, F. L., Shuster, M. D., 1982. Kalman filtering for spacecraft attitude estimation. In: Proc. AIAA 20th Aerospace Sciences Meeting. pp. 1-16.

Loría, A., Panteley, E., Popović, D., Teel, A. R., 2005. A nested Matrosov theorem and persistency of excitation for uniform convergence in stable nonautonomous systems. IEEE Trans. Automat. Contr. 50 (2), 183-198.

Mahony, R., Hamel, T., Pflimlin, J.-M., 2008. Nonlinear complementary filters on the Special Orthogonal Group. IEEE Trans. Automat. Contr. 53 (5), 1203-1218.

Mahony, R., Hamel, T., Trumpf, J., Lageman, C., 2009. Nonlinear observers on $\mathrm{SO}$ (3) for complementary and compatible measurements: A theoretical study. In: Proc. IEEE Conf. Dec. Contr. Shanghai, China, pp. 6407-6412.

Maybeck, P. S., 1979. Stochastic Models, Estimation, and Control, Volume 1. Vol. 141 of Mathematics in Science and Engineering. Academic Press.

Panteley, E., Loría, A., 2001. Growth rate conditions for uniform asymptotic stability of cascaded time-varying systems. Automatica 37 (3), 453-460.

Phillips, R., Schmidt, G., 1996. GPS/INS integration. AGARD Lecture Series: System Implications and Innovative Applications of Satellite Navigation 207, 9.1-9.18.

Salcudean, S., 1991. A globally convergent angular velocity observer for rigid body motion. IEEE Trans. Automat. Contr. 36 (12), 1493-1497.

Shuster, M. D., Oh, S. D., 1981. Three-axis attitude determination from vector observations. J. Guid. Contr. Dynam. 4 (1), 70-77.

Thienel, J. K., Sanner, R. M., 2003. A coupled nonlinear spacecraft attitude controller and observer with an unknown constant gyro bias and gyro noise. IEEE Trans. Automat. Contr. 48 (11), 2011-2015.

Vik, B., Fossen, T. I., 2001. A nonlinear observer for GPS and INS integration. In: Proc. IEEE Conf. Dec. Contr. Orlando, FL, pp. 2956-2961.

\section{Appendix A. PARAMETER PROJECTION}

Let the set of possible parameters be defined by $\mathcal{B}_{g}:=\left\{b_{g} \in\right.$ $\left.\mathbb{R}^{3} \mid \mathcal{P}\left(b_{g}\right) \leq 0\right\}$, where $\mathcal{P}: \mathbb{R}^{3} \rightarrow \mathbb{R}$ is a smooth, convex function. Let $\mathcal{B}_{g}^{0}$ denote the interior of $\mathcal{B}_{g}$, and let $\hat{\mathcal{B}}_{g}$ be defined by $\hat{\mathcal{B}}_{g}=\left\{\hat{b}_{g} \in \mathbb{R}^{3} \mid \mathcal{P}\left(\hat{b}_{g}\right) \leq \delta\right\}$, where $\delta$ is a small positive number, making $\hat{\mathcal{B}}_{g}$ a slightly larger superset of $\mathcal{B}_{g}$. Then $\operatorname{Proj}\left(\hat{b}_{g},-k_{I} \sigma\right)=p\left(\hat{b}_{g},-k_{I} \sigma\right)\left(-k_{I} \sigma\right)$, where

- $p\left(\hat{b}_{g},-k_{I} \sigma\right)=I$ if $\hat{b}_{g} \in \mathcal{B}_{g}^{0}$ or $\nabla \mathcal{P}^{\top} \cdot\left(-k_{I} \sigma\right) \leq 0$.

- $p\left(\hat{b}_{g},-k_{I} \sigma\right)=I-c\left(\hat{b}_{g}\right) \nabla \mathcal{P} \nabla \mathcal{P}^{\top} /\|\nabla \mathcal{P}\|^{2}$ if $\hat{b}_{g} \in \hat{\mathcal{B}}_{g} \backslash \mathcal{B}_{g}^{0}$ and $\nabla \mathcal{P}^{\top} \cdot\left(-k_{I} \sigma\right)>0$,

where $\nabla \mathcal{P}^{\top}$ is the gradient of $\mathcal{P}$ and $c\left(\hat{b}_{g}\right)=\min \left\{1, \mathcal{P}\left(\hat{b}_{g}\right) / \delta\right\}$.

\section{Appendix B. PROOFS}

Proof of Theorem 1 Let $M$ be a bound on $\left\|\tilde{b}_{g}\right\|$ on the compact set $\tilde{\mathcal{B}}_{g}=\left\{\tilde{b}_{g} \mid b_{g} \in \mathcal{B}_{g}, \hat{b}_{g} \in \hat{\mathcal{B}}_{g}\right\}$. We start by defining $\ell>0$ such that $\ell<\min \left\{1 /\left(2 k_{I}\right), c_{\mathrm{obs}}^{2} \varepsilon^{2} /\left(12 n M+8 k_{I} n\right)\right\}$. 
Next, define $a=c_{\mathrm{obs}}^{2} \varepsilon^{2}-\ell\left(12 n M+8 k_{I} n\right)$ (note that $a>0$ due to the definition of $\ell$ ) and let $\bar{k}_{P}=\max \left\{M /\left(c_{\mathrm{obs}}^{2} \varepsilon^{2}(1-\right.\right.$ $\left.\left.\left.\varepsilon^{2}\right)\right),\left((1+2 \ell \bar{\omega})^{2}+4 \ell^{2} M^{2} \varepsilon^{2}\right) /\left(4 a \ell \varepsilon^{2}\right)\right\}$. Using unit quaternions the error dynamics (8) becomes

$$
\begin{aligned}
\dot{\tilde{s}} & =\frac{1}{2} \tilde{r}^{\top} R\left(\tilde{b}_{g}+\sigma\right), \\
\dot{\tilde{r}} & =-\frac{1}{2}(\tilde{s} I-S(\tilde{r})) R\left(\tilde{b}_{g}+\sigma\right), \\
\dot{\tilde{b}}_{g} & =-\operatorname{Proj}\left(\hat{b}_{g},-k_{I} \sigma\right) .
\end{aligned}
$$

We know that $\hat{b}_{g}(0) \in \hat{\mathcal{B}}_{g}$. Due to the projection, it therefore follows that for all $t \geq 0, \hat{b}_{g}(t) \in \hat{\mathcal{B}}_{g}$, and hence $\tilde{b}_{g}(t) \in \tilde{\mathcal{B}}_{g}$. Consider the derivative of $V$ defined in (5):

$$
\dot{V} \leq-\tilde{s} \tilde{r}^{\top} R \tilde{b}_{g}-k_{P} c_{\mathrm{obs}}^{2} \tilde{s}^{2}\left(1-\tilde{s}^{2}\right) \leq M-k_{P} c_{\mathrm{obs}}^{2} \tilde{s}^{2}\left(1-\tilde{s}^{2}\right) .
$$

Using the above definition of $\bar{k}_{P}$, we see that $|\tilde{s}|=\varepsilon \Longrightarrow \dot{V}<$ 0 . This implies that $V$ is strictly decreasing whenever $|\tilde{s}|=\varepsilon$, which in turn implies that $|\tilde{s}|$ is strictly increasing. It follows from $|\tilde{\mathcal{S}}(0)| \geq \varepsilon$ and continuity of the solutions that $|\tilde{s}|$ can never become smaller than $\varepsilon$, and we therefore assume $|\tilde{s}| \geq \varepsilon$ in the remainder of the analysis.

Consider the Lyapunov function candidate

$$
W\left(\tilde{r}, \tilde{s}, \tilde{b}_{g}\right)=V+2 \ell \tilde{s} \tilde{r}^{\top} R \tilde{b}_{g}+\frac{\ell}{2 k_{I}} \tilde{b}_{g}^{\top} \tilde{b}_{g} .
$$

We have $W \geq\|\tilde{r}\|^{2}-2 \ell\|\tilde{r}\|\left\|\tilde{b}_{g}\right\|+\frac{\ell}{2 k_{l}}\left\|\tilde{b}_{g}\right\|^{2}$, where we have used the fact that $|\tilde{s}| \leq 1$. This quadratic expression is positive definite with respect to $\tilde{r}$ and $\tilde{b}_{g}$ due to the bound on $\ell$ described above. Moreover, $W \leq\|\tilde{r}\|^{2}+2 \ell\|\tilde{r}\|\left\|\tilde{b}_{g}\right\|+$ $\frac{\ell}{2 k_{I}}\left\|\tilde{b}_{g}\right\|^{2}$. It follows that there exist positive constants $\alpha_{1}$ and $\alpha_{2}$ such that $\alpha_{1}\left\|\left(\tilde{r}, \tilde{b}_{g}\right)\right\|^{2} \leq W \leq \alpha_{2}\left\|\left(\tilde{r}, \tilde{b}_{g}\right)\right\|^{2}$.

For the time derivative of $W$ we calculate

$$
\begin{aligned}
\dot{W} \leq & -\tilde{s} \tilde{r}^{\top} R \tilde{b}_{g}-k_{P} c_{\mathrm{obs}}^{2} \tilde{s}^{2}\left(1-\tilde{s}^{2}\right)+\ell \tilde{r}^{\top} R \tilde{b}_{g} \tilde{r}^{\top} R \tilde{b}_{g} \\
& +\ell \tilde{r}^{\top} R \sigma \tilde{r}^{\top} R \tilde{b}_{g}-\ell \tilde{s}^{2} \tilde{b}_{g}^{\top} \tilde{b}_{g} \\
& -\ell \sigma^{\top} R^{\top}\left(\tilde{s}^{2} I+\tilde{s} S(\tilde{r})\right) R \tilde{b}_{g}+2 \ell \tilde{s} \tilde{r}^{\top} R S(\omega) \tilde{b}_{g} \\
& -2 \ell \tilde{s} \tilde{r}^{\top} R \operatorname{Proj}\left(\hat{b}_{g},-k_{I} \sigma\right)-\frac{\ell}{k_{I}} \tilde{b}_{g}^{\top} \operatorname{Proj}\left(\hat{b}_{g},-k_{I} \sigma\right) \\
\leq & \|\tilde{r}\|\left\|\tilde{b}_{g}\right\|-k_{P} c_{\mathrm{obs}} \tilde{s}^{2}\|\tilde{r}\|^{2}+\ell\|\tilde{r}\|^{2}\left\|\tilde{b}_{g}\right\|^{2}-\ell \tilde{s}^{2}\left\|\tilde{b}_{g}\right\|^{2} \\
& -\ell \sigma^{\top} R^{\top}\left(\left(1-\|\tilde{r}\|^{2}\right) I+\tilde{s} S(\tilde{r})-\tilde{r} \tilde{r}^{\top}\right) R \tilde{b}_{g} \\
& +2 \ell \bar{\omega}\|\tilde{r}\|\left\|\tilde{b}_{g}\right\|+2 \ell k_{I}\|\tilde{r}\|\|\sigma\|+\ell \sigma^{\top} \tilde{b}_{g} \\
= & \|\tilde{r}\|\left\|\tilde{b}_{g}\right\|-k_{P} c_{\mathrm{obs}} \tilde{s}^{2}\|\tilde{r}\|^{2}+\ell\|\tilde{r}\|^{2}\left\|\tilde{b}_{g}\right\|^{2}-\ell \tilde{s}^{2}\left\|\tilde{b}_{g}\right\|^{2} \\
& -\ell \sigma^{\top} R^{\top}\left(-\|\tilde{r}\|^{2} I+\tilde{s} S(\tilde{r})-\tilde{r} \tilde{r}^{\top}\right) R \tilde{b}_{g} \\
& +2 \ell \bar{\omega}\|\tilde{r}\|\left\|\tilde{b}_{g}\right\|+2 \ell k_{I}\|\tilde{r}\|\|\sigma\|,
\end{aligned}
$$

where we have used the properties that $\left\|\operatorname{Proj}\left(\hat{b}_{g},-k_{I} \sigma\right)\right\| \leq$ $k_{I}\|\sigma\|$ and $-\tilde{b}_{g}^{\top} \operatorname{Proj}\left(\hat{b}_{g},-k_{I} \sigma\right) \leq k_{I} \tilde{b}_{g}^{\top} \sigma$ (Krstić et al., 1995,
Lemma E.1). Note that $\|\sigma\|=\|R \sigma\|$ and

$$
\|R \sigma\|=2\left\|\sum_{j=1}^{n} k_{j} S\left(v_{j}^{i}\right)\left(\tilde{s} S(\tilde{r})+S(\tilde{r})^{2}\right) v_{j}^{i}\right\| \leq 4 k_{P} n\|\tilde{r}\| .
$$

Using this bound, as well as $|\tilde{s}| \geq \varepsilon$, we obtain

$$
\begin{aligned}
\dot{W} \leq & \|\tilde{r}\|\left\|\tilde{b}_{g}\right\|-k_{P} c_{\mathrm{obs}}^{2} \varepsilon^{2}\|\tilde{r}\|^{2}+\ell\|\tilde{r}\|^{2}\left\|\tilde{b}_{g}\right\|^{2}-\ell \varepsilon^{2}\left\|\tilde{b}_{g}\right\|^{2} \\
& +4 \ell k_{P} n\|\tilde{r}\|\left(2\|\tilde{r}\|^{2}+\|\tilde{r}\|\right)\left\|\tilde{b}_{g}\right\|+2 \ell \bar{\omega}\|\tilde{r}\|\left\|\tilde{b}_{g}\right\| \\
& +8 \ell k_{I} k_{P} n\|\tilde{r}\|^{2} \\
\leq & \|\tilde{r}\|\left\|\tilde{b}_{g}\right\|-k_{P} c_{\mathrm{obs}}^{2} \varepsilon^{2}\|\tilde{r}\|^{2}+\ell M^{2}\|\tilde{r}\|^{2}-\ell \varepsilon^{2}\left\|\tilde{b}_{g}\right\|^{2} \\
& +12 \ell k_{P} n M\|\tilde{r}\|^{2}+2 \ell \bar{\omega}\|\tilde{r}\|\left\|\tilde{b}_{g}\right\|+8 \ell k_{I} k_{P} n\|\tilde{r}\|^{2} \\
= & -\left(k_{P} a-\ell M^{2}\right)\|\tilde{r}\|^{2}-\ell \varepsilon^{2}\left\|\tilde{b}_{g}\right\|^{2} \\
& +(1+2 \ell \bar{\omega})\|\tilde{r}\|\left\|\tilde{b}_{g}\right\| .
\end{aligned}
$$

Using the definition of $\bar{k}_{P}$, it is straightforward to show that this expression is negative definite with respect to $\tilde{r}$ and $\tilde{b}_{g}$; that is, there exists an $\alpha_{3}>0$ such that $\dot{W} \leq-\alpha_{3}\left\|\left(\tilde{r}, \tilde{b}_{g}\right)\right\|^{2}$, and thus there is an $\alpha>0$ such that $\dot{W} \leq-\alpha W$. Using the comparison lemma (Khalil, 2002, Lemma 3.4), we can therefore conclude that there exist positive constants $K$ and $\lambda$ such that for all $t \geq 0,\left\|\left(\tilde{r}(t), \tilde{b}_{g}(t)\right)\right\| \leq K \mathrm{e}^{-\lambda t}\left\|\left(\tilde{r}(0), \tilde{b}_{g}(0)\right)\right\|$. Noting that $\|I-\tilde{R}\|=\sqrt{8}\|\tilde{r}\|$, we therefore have $\|(I-$ $\left.\tilde{R}(t), \tilde{b}_{g}(t)\right)\left\|\leq \sqrt{8} K \mathrm{e}^{-\lambda t}\right\|\left(I-\tilde{R}(0), \tilde{b}_{g}(0)\right) \|$.

Proof of Theorem 2 Let $M$ be a bound on $\|\phi\|$, and consider the Lyapunov function (inspired by Loría, Panteley, Popović, and Teel, 2005)

$$
V(t, \tilde{\theta})=\frac{1}{2} \tilde{\theta}^{\top}\left(\Gamma^{-1}-\ell \int_{t}^{\infty} \mathrm{e}^{t-\tau} \phi(\tau) \phi(\tau)^{\top} \mathrm{d} \tau\right) \tilde{\theta},
$$

where $\ell<\min \left\{\lambda_{\min }\left(\Gamma^{-1}\right) / M^{2}, 2 \mathrm{e}^{-T} \varepsilon /\left(M^{6} \lambda_{\max }^{2}(\Gamma)+\mathrm{e}^{-T} \varepsilon\right)\right\}$. We have $V \leq \frac{1}{2} \lambda_{\max }\left(\Gamma^{-1}\right)\|\tilde{\theta}\|^{2}$ and $V \geq \frac{1}{2}\left(\lambda_{\min }\left(\Gamma^{-1}\right)-\ell M^{2}\right)\|\tilde{\theta}\|^{2}$, which is positive definite due to the bound on $\ell$. Furthermore,

$$
\begin{aligned}
\dot{V}= & -\tilde{\theta}^{\top} \phi \phi^{\top} \tilde{\theta}+\ell \tilde{\theta}^{\top} \int_{t}^{\infty} \mathrm{e}^{t-\tau} \phi(\tau) \phi(\tau)^{\top} \mathrm{d} \tau \Gamma \phi \phi^{\top} \tilde{\theta} \\
& +\frac{1}{2} \ell \tilde{\theta}^{\top} \phi \phi^{\top} \tilde{\theta}-\frac{1}{2} \ell \tilde{\theta}^{\top} \int_{t}^{\infty} \mathrm{e}^{t-\tau} \phi(\tau) \phi(\tau)^{\top} \mathrm{d} \tau \tilde{\theta} \\
\leq & -\left|\phi^{\top} \tilde{\theta}\right|^{2}+\ell M^{3} \lambda_{\max }(\Gamma)\|\tilde{\theta}\|\left|\phi^{\top} \tilde{\theta}\right| \\
& +\frac{1}{2} \ell\left|\phi^{\top} \tilde{\theta}\right|^{2}-\frac{1}{2} \ell \tilde{\theta}^{\top} \int_{t}^{t+T} \mathrm{e}^{-T} \phi(\tau) \phi(\tau)^{\top} \mathrm{d} \tau \tilde{\theta} \\
\leq & -\left(1-\frac{1}{2} \ell\right)\left|\phi^{\top} \tilde{\theta}\right|^{2}+\ell M^{3} \lambda_{\max }(\Gamma)\|\tilde{\theta}\|\left|\phi^{\top} \tilde{\theta}\right| \\
& -\frac{1}{2} \ell \mathrm{e}^{-T} \varepsilon\|\tilde{\theta}\|^{2} .
\end{aligned}
$$

This is a quadratic expression with respect to $\|\tilde{\theta}\|$ and $\left|\phi^{\top} \tilde{\theta}\right|$, which is easily shown to be negative definite by using the bound on $\ell$. Once more the result follows from using the comparison lemma (Khalil, 2002, Lemma 3.4). 